\title{
Register rerum ad Vol. 8
}

\section{Bearbeitet von Wilhelm Baumann}

Accessory male generative organs of the rat, experimental study, 343 Acid phosphatases in prostatic carcinoma, 55 Arthus-Phänomen, Nierenblutung, 65 Bassinet, structure neuro-musculaire, 272 Bladder, electromagnetic stimulation, 32 Blase, transkutane Reizung bei Lähmung, 32 Blasenscheidenfisteln, Behandlung, 38 Canal deferent, atrophie congénitale, 45 Canal de Wolff, separation de Turetère primitif, 117 Carcinome de la prostate, phosphatases, 55 -, traitement par le diéthylstilboestrol phosphorylé, 55 Carcinome du duodenum, compression de Turetère, 215 Carcinome du pancreas, compression de Гuretère, 215 Circulation rénale, Гinfluence de ГEphédrine, 142 Cloaca, hypospadias and aplasia of the kidney, 317 Collision-tumour in the prostatic area, 256 Contracted kidney after injury, 193 Cord bladder, stimulation electromagnetic, 32 Cystectomie sub-totale, implantation de Turetère, 1 Cystitis. Antibiotica, 158, 174 Cysto-pyélite chronique, bactériologie et antibiotiques, 158, 174 Decapsulation et hématurie rénale, 65

et néphroptose, 65 Dekapsulation bei Néphroptose, 65

bei Nierenblutung, 65 Ductus deferens, Agenesie, 45

Duodenal carcinoma, compression of the ureter, 215

Ephedrin, Einfluß auf die Nierendurch-blutung, 142

Ephedrine, influence of the renal circulation, 142

Epididymite chronique, biopsie trans-cutanée, 152

Fistules vésico-vaginales, traitement, 38

Geschlechtsdrüsen, sekundäre, experi-mentelle Studien an der Ratte, 343

Haematuria renalis, 65

Harnleiterimplantation, 1

Harnwegsinfektionen, Antibiotika, 158, 174

-, bakterielle Untersuchungen, 158, 174

Hématurie rénale allergique, 65

Hémodynamique rénale et la pyélo-néphrite chronique, 177

Hemorrhage, renal, 65

Hochdruck bei posttraumatischen Schrumpfnieren, 193

Hydronephrose et carcinome prostatique, 103

- $\quad$ et hypertrophie prostatique, 103Hydronephrosis in prostatic adenoma, 
intraveineuse, 103

Hypoxie und Nierenblutung, 65

Hypospadias, aplasie rénale, persistance du cloaque, 317

Hypospadias, cloaca and aplasia of the kidney, 317

Hypospadie, Nierenaplasie und Kloake, 317

Ileo-cysto-plasty, 1

Implantation de $\Gamma$ uretère, 1

Infection du tractus urinaire, antibiotiques, 158, 174

VII/1959

, bactériologie, 158, 174

Register rerum

375

Kidney, aplasia with hypospadias and cloaca, 317

-, congenital multicystic dysplasia, 204

-, consequences of blunt traumatism, 193

-, contracted after injury, 193

-, decapsulation and hematuria, 65

-, influence of Ephedrine on the circulation, 142

-, nephropexy and hematuria, 65

-, parapyelic cysts, 228

-, partial resection, 126

-, tuberculous, indications for conservative operation, 126

Kloake, Hypospadie-Nierenaplasie, 317

Kollisionstumor im Bereich der Pro-stata, 256

Kystes parapyéliques, 228

Malformation urogénitale complexe, 317

Nebenhoden, Biopsie, 152

Nephropexy and renal hematuria, 65

Néphroptose et hématurie, 65

Neomycin bei Harnwegsinfektionen, 174

Neomycin in urinary tract infections, 174

Niere, Aplasie mit Kloake und Hypo-spadie, 317

-, Blutung, 65

-, Dekapsulation und Hämaturie, 65

-, Einfluß des Ephedrins auf die Durch-blutung, 142

-, konservative Operationsindikationen bei Tuberkulose, 126

-, angeborene multicystische Dysplasie, 204

-, Nephropexie und Hämaturie, 65

-, Nierenbeckencysten, 228

-, Ptose, 65

-, Spätfolgen nach stumpfen Traumen, 193

-, posttraumatische Schrumpfnieren, 193

Nierenbecken, pharmakologische Struk-turanalysen, 272

Nierenblutung, 65

Nierenresektion, partielle, 126 
Nierentraumen, stumpfe, Spätfolgen, 193

Organes sexuels secondaires du rat, observations expérimentales, 343

Ouraque, tumeur, 25

Oxalosis, 234

Pancreatic carcinoma, compression of the ureter, 215

Parapyelic cysts, 228

Penis, dermal plastic operation, 12

Penis, plastie cutanée, 12

Periureteritis fibrosa und Tumor, 215

Phalloplastik, 12

Phosphatase, saure, bei Prostata-Carcinom, 55

Prostata, Carcinosarcom, 256

- $\quad$ Harnstau bei Adenom, 103

-, Harnstau bei Carcinom, 103 Prostata-Carcinom, Behandlung mit

phosphoryliertem Diäthylstilböstrol,

55 -, saure Phosphatase, 55 -, Harnstau, 103 Prostatahypertrophie, Ausscheidungs-

urogramm, 103 Prostate, carcinoma-sarcoma, 256 Prostate, carcinome, hydronéphrose,

103 -, -, phosphatases, 55 -, -, traitement par le stilboestrol, 55 -, hypertrophie, hydronéphrose,

103 Prostatectomie, urographie intra-

veineuse, 103 Prostatectomy, excretory urography,

103 Prostatektomie, Pyelogramm prae- und

postoperativ, 103 Prostatic carcinoma, acid phosphatases,

55

, hydronephrosis, 103

, treatment with phosphorylated di-

ethylstilbestrol, 55 Prostatic hypertrophy, excretory uro-

gramm, 103

, hydronephrosis, 103

Pyelocystitis, Antibiotica, 174 Pyélonéphrite chronique, le traitement

prolongé, 20, 289 tension artérielle, 177

Thémodynamique rénale, 177

l'épreuve de resistance, 20 -, tension artérielle, 177 Pyelonephritis, extended therapy, 20,

289 Pyelonephritis, Hypertonie, 177 -, Langzeitbehandlung, 20, 289 -, renale Hämodynamik,

177 -, Resistenzbestimmungen, 20

376 Regist.

rerum VIII/1959

Rein, aplasie avec hypospadias et per-

sistance du cloaque, 317 -, contracté posttraumatique, 193 -, decapsulation et hématurie, 65 -, dysplasie polykystique congénitale,

204 -, indications opératoires conservatrices

de la tuberculose, 126 -, kystes parapyéliques, 228 -, Гinfluence de ГEphédrine sur la

circulation, 142 -, néphropexie et hématurie, 65 -, ptose et hématurie, 65 -, resection partielle, 126 -, séquelles des traumatismes fermés, 193 Renal circulation, influence of Ephedrine, 142 - pelvis, pharmacologic analysis of structure, 272 Resection partielle du rein, 126

Retroperitoneal carcinoma, differential 
diagnosis, 215 Samenleiter, Sterilität durch Agenesie,

45 Sarco-épithélioma de la region prosta-

tique, 256 Schrumpfnieren, posttraumatische, 193 Sterilität, Agenesie des ductus deferens, 45 -, Nebenhodenbiopsie, 152 Sterility by absence of ductus deferens,

45 -, biopsy of the epididymis, 45 Streptomycin bei Harnwegsinfektionen,

174 Streptomycin in urinary tract infections,

174 Tetracyclin bei Harnwegsinfektionen,

174 Tetracyclin in urinary tract infections,

174 Tuberkulose der Nieren, konservative

Operationsindikationen, 126

Tuberculose rénale, Гindication opéra-toire conservatrice, 126

Tuberculose urogenitale, la biopsie de Гépididyme, 152

Tuberculous kidney, indications for conservative operation, 126

Urachus tumor, 25

Ureter, Abspaltung des primitiven Ureters vom Wolffschen Gang, 117

-, Kompression durch Tumorinvasion, 215

-, Erweiterung bei Prostatahypertro-phie, 103

Ureter, compression following neopla-stic invasion, 215

-, implantation, 1

-, separation from the Wolffian duct, 117

Uretère, compression par invasion tu-morale, 215

-, primitif, separation du canal de Wolff, 117

Urinary tract infections, antibiotic drugs, 174 , bacteriology, 174

Urogenital neoplasia, Csaba-Törö reaction, 336

tractus, malformation, 317

tuberculosis, biopsy of the epididymis, 152

Urogenitalapparat, kombinierte Miß-bildung, 317

Urogenitalgeschwülste, Csaba-Törö-Reaktion, 336

Urogenitaltuberkulose, Nebenhodenbiopsie, 152

Urolithiasis, Oxalosis, 234

Vessie médullaire, excitation transcu-tanée, 32

W. Wolffian duct

separation from the primitive ureter

117

Wolffscher Gang

Abspaltung des primitiven Ureters

117 